\title{
Philosophiques
}

\section{Langage ordinaire et langage abstrait chez Guillaume d'Occam}

\section{Claude Panaccio}

Volume 1, numéro 2, octobre 1974

URI : https://id.erudit.org/iderudit/203012ar

DOI : https://doi.org/10.7202/203012ar

Aller au sommaire du numéro

Éditeur(s)

Société de philosophie du Québec

ISSN

0316-2923 (imprimé)

1492-1391 (numérique)

Découvrir la revue

Citer cet article

Panaccio, C. (1974). Langage ordinaire et langage abstrait chez Guillaume d’Occam. Philosophiques, 1(2), 37-60. https://doi.org/10.7202/203012ar

\section{Résumé de l'article}

Utilisant surtout le traité De Sacramento Altaris, l'auteur essaie de montrer que, bien que l'opposition langage ordinaire/langage philosophique soit effectivement présente chez Guillaume d'Occam, sa critique logique du discours abstrait n'a cependant rien à voir, quoi qu'en pensent certains commentateurs, avec la philosophie contemporaine du langage ordinaire, puisqu'elle trouve son critère et sa norme dans un discours mental, naturel, universel et prélinguistique. De façon plus générale, l'article vise à mettre au jour certains présupposés épistémologiques qui doivent être dépassés pour qu'une « philosophie du langage ordinaire » devienne historiquement possible.
Ce document est protégé par la loi sur le droit d'auteur. L'utilisation des services d’Érudit (y compris la reproduction) est assujettie à sa politique d'utilisation que vous pouvez consulter en ligne.

https://apropos.erudit.org/fr/usagers/politique-dutilisation/ 


\title{
LANGAGE ORDINAIRE ET LANGAGE ABSTRAIT CHEZ GUILLAUME D'OCCAM
}

\author{
par Claude Panaccio
}

On s'accorde généralement à considérer Guillaume d'Occam comme l'un des plus grands penseurs du Moyen Âge. Par ailleurs, les travaux remarquables de plusieurs chercheurs contemporains, Philotheus Boehner et Ernest Moody en particulier ${ }^{1}$, ont largement contribué à mettre un terme aux rumeurs complètement fausses qui ont longtemps circulé à son sujet et selon lesquelles, par exemple, il était nominaliste, empiriste et sceptique. Malgré tout cela, Occam est encore très mal connu. L'édition critique de ses oeuvres complètes, actuellement en cours à l'Université St-Bonaventure, est loin d'être terminée; très peu de choses sont traduites en langues modernes, et l'occamisme se limite encore pour le public philosophique à la critique des universaux, à l'utilisation du « rasoir » et à la thèse de l'omnipuissance divine. Et certes ces éléments jouent effectivement un rôle important dans le système philosophico-théologique de ce Franciscain du XIVe siècle, mais ce qu'on connait très peu, c'est la place centrale occupée dans ce système par la logique comme méthode d'analyse du langage ${ }^{2}$.

La fameuse critique des universaux par exemple se présente le plus souvent comme la critique d'une utilisation impropre du discours, basée sur une confusion entre les différents

1. Cf. surtout : MOODY, E.A. The Logic of William of Ockbam, New York, Sheed and Ward, 1935 ; et BOEHNER, P. Collected articles on Ockbam, St-Bonaventure, Franciscan Institute Publications, 1958.

2. Bien que Moody, Boehner et plusieurs autres aient continuellement insisté sur ce point. 
modes de signification et sur la croyance selon laquelle les termes abstraits comme «humanité», «animalité », « essence», "existence », etc... se comportent logiquement de la même manière que les termes concrets comme «homme», «animal», «individu» etc... La question qui fait l'objet du présent article concerne les critères de cette critique du langage abstrait. L'une des grandes utilités de la logique, au dire d'Occam, est de permettre la reconnaissance du sens propre d'un discours et sa discrimination d'avec l'usage impropre et métaphorique ${ }^{3}$. Mais d'où le discours tient-il cette proprietas ou cette virtus ? À quel étalon mesure-t-on la correction de l'usage linguistique? Je voudrais en particulier montrer qu'il est impossible de retrouver dans l'oeuvre d'Occam quelque recours que ce soit au «langage ordinaire» comme critère.

Cette thèse, si elle est accréditée, présente, à mon sens, un double intérêt :

1) sur un plan monographique d'abord, elle permet de cerner plus adéquatement la portée exacte de la logique occamiste en éliminant définitivement toute interprétation de cette logique en termes de "philosophie du langage ordinaire». Dans sa traduction du De Sacramento Altaris ${ }^{4}$, T. Bruce Birch rend systématiquement secundum proprietatem sermonis par «according to a property of common speech $»^{5}$. Or si, comme j'entends le montrer, c'est là une erreur, elle est grave, non seulement parce que l'expression est très fréquente chez Occam, mais surtout parce que cette fréquence est directement fonction de l'importance théo-

3. Cf. Expositionis in libros artis logicae prooemium, éd. par MOODY, E.A., St-Bonaventure, Franciscan Institute Publications, 1965, p. 4-5: «Circa quod sciendum quod istius scientiae sunt multae utilitates, inter quas una est facilitas discernendi inter verum et falsum (...) Secunda utilitas est promptitudo respondendi (..) Alia utilitas logicae est facilitas virutem sermonis et proprium modum loquendi percipiendi. Nam per istam artem faciliter scitur quid ab auctoribus de virtute sermonis profertur, quid non de virtute sermonis sed secundum usitatum modum loquendi vel secundum intentionem dicentis; quid dicitur proprie, quid metapborice.» (souligné par moi)

4. De Sacramento Altaris, texte latin et traduction anglaise par T.B. BIRCH, Iowa, The Lutheran Literary Board, 1930.

5. On trouve par exemple entre les pages 40 et 66 une vingtaine d'occurrences de cette expression. Toujours traduite de la même façon par Birch. 
rique de la proprietas sermonis comme critère de l'analyse logique du discours. Dans l'état actuel des choses, la traduction de Birch constitue l'une des rares versions en langue moderne d'un traité complet d'Occam, et par cette erreur d'apparence assez bénigne, elle contribue à propager une image sérieusement déformée du fonctionnement réel de la logique occamiste.

Plus récemment, dans son livre sur la logique de SaintAnselme, D.P. Henry, qui est pourtant un éminent médiéviste, tombe dans la même erreur en classant résolument Occam (avec Jean de Salisbury) parmi les philosophes médiévaux du langage ordinaire, qui selon lui s'opposaient à la tradition anselmienne de privilégier la forme logique sur l'usage grammatical :

La caractéristique la plus importante et la plus significative de l'oeuvre d'Occam, écrit-il, réside dans le fait que son attitude vis-à-vis l'opposition entre langage ordinaire et langage technique est à l'exact opposé de celle d'Anselme. Ainsi que nous l'avons vu, ce dernier situe la «propriété » et la signification per se du côté du discours technique, l'impropriété et la signification oblique du côté de l'usus loquendi; Occam au contraire s'efforce continuellement de montrer l'inutilité du langage technique, ou alors il le condamne comme faux ou comme dénué de sens au nom d'un critère de propriété qui pour lui se situe clairement du côté du langage ordinaire ${ }^{6}$.

Le fait qu'à l'appui de cette affirmation, Henry cite un long passage de la Summa logicae ${ }^{7}$, où Occam critique au nom de la proprietas sermonis un certain usage des termes abstraits, montre bien que ce sont précisément ces deux notions de proprietas ser-

6. The Logic of Saint Anselm, Oxford, Oxford University Press, 1967, p. 26: "The most important and significant feature of Ockham's work lies in the fact that his attitude to the contrast between ordinary and technical language is the polar opposite of that of Anselm. As we have seen, the latter sites 'propriety' and per se signification on the side of technical discourse, and impropriety and oblique signification on the side of usus loquendi; whereas Ockham unceasingly strives to make technical language pointless, or else condemns it as false or senseless by reference to the standpoint and criterion of a 'propriety' which for him clearly lies on the side of ordinary langage. $》$ (traduit par moi).

7. Cf. Summa logicae, pars prima, éd. par BOEHNER, P., St-Bonaventure. Franciscan Institute Publications, 1951, ch. 7, pp. 26-27. 
monis et de terminus abstractus qui sont ici en cause et que c'est sur elles que doit d'abord porter la discussion ${ }^{8}$.

2) sur un plan plus proprement philosophique, la thèse apparemment historique que je veux défendre ici à propos d'un auteur du XIVe siècle peut servir d'appoint à une hypothèse beaucoup plus générale, selon laquelle le recours au langage ordinaire comme critère de la validité logique n'est possible que dans un contexte épistémique particulier où se trouve dépassée la dissociation radicale du couple pensée/langage. Auquel cas l'erreur de Birch et de Henry serait en même temps un anachronisme! Sans aborder l'examen détaillé de cette hypothèse dans toute son ampleur, je voudrais en tout cas montrer que l'impossibilité pour Occam de conférer à l'usage ordinaire le statut d'une norme est liée dans son oeuvre à la position d'un discours mental universel et prélinguistique, par rapport auquel tous les «usages » oraux ou écrits ne sont que des sous-produits.

J'ai choisi d'adopter comme point de départ non pas la théorie explicite du discours abstrait, mais la pratique même de

8. Le caractère totalement erroné de l'interprétation d'Occam par Henry a déjà été souligné par John TRENTMAN ( Extraordinary Language and Medieval Logic 》), Dialogue, vol. VII, no 2, sept. 1968, pp. 286-291). Trentman, tout comme je le ferai moi-même ici, fonde sa critique sur la place importante occupée dans la logique occamiste par le langage mental considéré comme structure logique idéale. Il montre ainsi que Guillaume d'Occam est beaucoup plus proche d'Anselme que ne le croit Henry. Mais cette réponse, si pertinente soit-elle, demeure malheureusement incomplète, car si Trentman va droit au coeur du problème (la question du langage mental), il ne jette cependant aucune lumière nouvelle sur cela même qui a suscité l'erreur de Henry, la notion de proprietas sermonis et la critique du langage abstrait. Ce qu'il dit - très brièvement - de ces deux points est même plutôt trompeur, puisque : (a) il semble admettre avec Henry que la proprietas sermonis réside dans la conformité à l'usage ordinaire (cf. p. 290 : « his whole philosophical method involves constant comparison between what is said, surface structure, structure secumdum proprietatem sermonis, and the logical structure of an ideal language, which for him is mental language »); (b) il soutient cependant que la proprietas sermonis n'est pas nécessairement valorisée par Occan (cf. p. $290:$ : to say that sentences like 'Every man has humanity' offend verbal propriety is not to praise verbal propriety »). J'essaierai de montter pour ma part que la proprietas sermonis chez Occam est déterminée non par l'usage mais par la structure logique elle-même et que partant elle est bel et bien valorisée. 
sa critique telle qu'on la trouve dans un traité théologique typiquement médiéval, le De Sacramento Altaris (Traité de l'Euchatistie) dont je tirerai quelques exemples particulièrement significatifs pour mon propos. J'essaierai ensuite, en recourant cette fois aux textes plus théoriques consacrés à la distinction concret/ abstrait ${ }^{9}$, de montrer qu'il faut interpréter ces exemples à partir du primat accordé par Occam aux considérations d'ordre sémantique et que la théorie de la signification elle-même, loin de donner lieu à un relativisme linguistique, s'appuie sur l'existence naturelle d'un langage logique, idéal, universel et normatif. Je conclurai cette étude en proposant quelques remarques sur la façon dont l'occamisme ainsi compris s'insère de plain-pied dans la tradition de la métaphysique occidentale et exclut par principe toute philosophie du langage ordinaire.

\section{I - Le De Sacramento Altaris et la critique du discours abstrait.}

Il convient d'abord de présenter brièvement ce traité assez étrange qu'est le De Sacramento Altaris. Sa bizarrerie apparente réside en ceci que l'ouvrage tel que publié et traduit par Birch ${ }^{10}$

9. J'utiliserai surtout: Summa logicae, pars prima, ch. 5-9, pp. 16-33; et Quoldlibeta Septem, Strasbourg, 1491, Quodl. V. question 9.

10. A vrai dire, l'édition de Birch que j'utiliserai ici est sans doute quelque peu déficiente. D'abord elle présente comme les deux parties d'un même ouvrage ce que L. BAUDRY considère être deux traités distincts «Sur trois manuscrits occamistes 》, (Arcbives d'bistoire doctrinale et littéraire du Moyen Age, T.X, 1936. pp. 129-162); et bien que cette question ne ne soit pas encore définitivement résolue, l'opinion de Baudry semble actuellement prédominante (pour une discussion de ce problème, voir en particulier: BUESCHER, G. The Eucharistic Teacbing of Willian Ockbam, St-Bonaventure, Franciscan Institute Publications, 1950, pp. xii-xiv; et BRAMPTON, C.K. "A note on the manuscript tradition of Ockham's tractatus 'De Quantitate' », Archivum Franciscanum bistoricum, vol. 57, 1954, pp. 383-391). Par ailleurs, Baudry, dans le même article, relève dans le texte édité par Birch, plusieurs inexactitudes. Mais ces déficiences sont de peu d'importance pour l'utilisation de ce texte que je propose ici. Il ne s'agit que d'en tirer quelques exemples d'analyses du discours abstrait, et de ce point de vue, il n'est pas très pertinent de savoir s'il y a là un seul traité ou deux, puisque de toute façon leur problématique et leur date de composition sont très rapprochées l'une de l'autre (cf. BAUDRY, L. Guillaume d'Occam, sa vie, ses oeuvres, ses idées sociales et politiques, T.I. Paris, Vrin, 1950, pp. $89-90$; et BRAMPTON, C.K. «Guillaume d'Ockham et la date probable de ses opuscules sur l'Eucharistie », 
porte finalement plus sur le statut sémantique des termes exprimant la «quantité »" que sur l'Eucharistic proprement dite.

La première partie du traité ${ }^{12}$ consiste exclusivement en une analyse des relations entre des termes tels que «point», «ligne», «surface », «corps», «quantité », et soulève les questions suivantes auxquelles il est toujours répondu par la négative : un point est-il une chose réelle distincte de la quantité ${ }^{13}$ ? Une ligne et une surface sont-elles des choses réelles distinctes l'une de l'autre et distinctes d'un corps ${ }^{14}$ ? Un corps étendu est-il une chose en soi (absoluta res) réellement distincte de la substance et de la qualité ${ }^{15}$ ? La deuxième partie elle-même ${ }^{16}$, intitulée pourtant «De corpore Christi», accorde une importance énorme (vingt-sept chapitres sur quarante et un) à un examen de la notion de quantité et de ses liens avec les notions de substance et de qualité.

Pour saisir la pertinence de ces discussions dans un traité de l'Eucharistie, il faut les situer dans le cadre de la problématique traditionnelle de la transsubstantiation: qu'est-ce exactement qui est conservé et qu'est-ce qui ne l'est pas dans la transformation du pain en corps du Christ? La réponse d'Occam - assez orthodoxe - est exposée rapidement dans les premiers chapitres de la deuxième partie: la substance du pain se transforme en substance du corps du Christ et par conséquent disparaît en tant que substance particulière, ce qui n'empêche pas ses qualités de sub-

Etudes franciscaines, 14, 1964, pp. 77-88.) Il parait donc plus commode pour mon propos de suivre l'édition Birch et de parler d'une "première partie» (qui correspondrait au De Sacramento Altaris proprement dit, également appelé le De Quantitate) et d'une «deuxième partie» (qui correspondrait à un traité De Corpore Christi). Par ailleurs, les incorrection; relevées par Baudry ne concernent en tien les exemples que je citerai ici. Je référerai dorénavant à cet ouvrage par le sigle S.A.

11. Il faut entendre par «quantité» soit le continu (i-e surtout le langage de l'étendue: point, ligne, surface, etc...), soit le discontinu dénombrable (cf. In librum praedicamentorum Aristotelis, éd. Bologne, 1496, chap. 10). Dans S.A. il est uniquement question du premier type de quantité.

12. S.A., pp. 2-156.

13. Id., pp. 6-82.

14. Id., pp. 82-92.

15. Id., pp. 92-156.

16. Id., pp. $158-500$. 
sister (dans l'ontologie occamiste, la qualité est séparable de la substance, au moins par intervention divine) sans devenir pour autant les qualités du corps du Christ.

À partir de là, ce qui intéresse surtout notre auteur, c'est de montrer que toutes les difficultés rencontrées par ses contemporains ${ }^{17}$ à expliquer comment un si grand corps peut tenir en un lieu si petit (l'hostie) ressortissent à une incompréhension de la "quantité » qu'ils prennent pour une chose réelle mais qui néanmoins n'est pas vraiment distincte de la substance et de la qualité, puisqu'elle n'est rien d'autre qu'une façon différente de signifier les mêmes réalités.

C'est précisément cette longue discussion du langage de l'étendue qui rend le $D e$ Sacramento Altaris pertinent à notre propos. On y saisit sur le vif de leur fonctionnement les instruments d'analyse sémantique dont Occam fait ailleurs la théorie, et on y repère en particulier de nombreux exemples de critique des modes impropres de parler, parmi lesquels je choisirai exprès ceux qui paraissent les plus susceptibles d'être interprétés en ter-

17. Occam semble avoir écrit le De Corpore Christi et le De Sacranento Alitaris pour répondre aux nombreuses accusations d'inorthodoxie que lui avaient values les passages de son Commentaire des Sentences consacrés à l'Eucharistie (cf. BAUDRY, L. «Sur trois manuscrits occamistes》, et surtout BUESCHER, G. The Eucharistic teaching of William of Ockbam, pp. xviii-xxvii). Il s'oppose principalement aux interprétations réalistes de la quantité fournies par certains de ses contemporains (sur le problème de leur identification, voir MOODY, E.A. «Ockham and Aegidius of Rome », Franciscan Studies, T. 9, 1949, pp. 417-442 ; MAIER, Anneliese, «Zu einigen Problemon der Ockhamforschung》, Archivum Franciscanum Historicum, T. 46, pp. 161-194.; BRAMPTON, C.K., «A note on Auriol, Ockham and Ms Borghese $329 »$, Gregorianum, T. 41, 1960, pp. 713-716), mais il reconnait aisément lui-même que l'importance énorme qu'il accorde aux questions concernant la quantité ne se retrouve pas chez les autorités théologiques ( Unde quamvis diligenter legerim totum tractatum Magistri Sententiarum de Eucharistia et totum tractatum, qui ponitur in iure canonico de eadem materia una cum glossis ct aliis doctoribus, sicut Hostiensem et Innoncentium et alios, tamen non inveni quod faciunt mentionem de quantitate etiam negando eam, nisi in tribus locis (...) In pluribus locis non memini me legisse quod Sancti vel Doctores authentici tractantes de Sacramento Eucharistiae aliquid tangunt de quantitate $\gg$, p. 276-278). C'est seulement pour répondre aux objections et pour résoudre les difficultés rencontrées par ses contemporains qu'il aborde lui-même ce problème avec tant d'insistance. 
mes de «philosophie du langage ordinaire», ceux où Occam opère la critique du discours abstrait.

Il faut d'abord admettre que, bien qu'elle se rencontre rarement, l'opposition explicite entre le langage ordinaire et le langage philosophique est bel et bien présente chez Occam. Ainsi, dans la première partie du De Sacramento Altaris, après avoir consacré quelques lignes à la façon d'interpréter les « dictos philosophorum et sanctorum », il commence le paragraphe suivant par les mots : «Immo etiam (...) vulgaris et communis modus loquendi... ${ }^{18}$, qu'on peut à juste titre traduire par : «En outre même la façon ordinaire et commune de parler... ». De même, le chapitre huit de la première partie de la Summa logicae oppose incidemment la vulgaris locutio aux dictis pbilosophorum et sanctorum:

Bien que le langage ordinaire ne recoure que rarement ou même jamais à ces termes abstraits dont la signification équivaut à celle de plusieurs mots, cependant on en rencontre souvent l'usage dans les discours des philosophes ou des saints. ${ }^{19}$

Aussi doit-il être clair que l'anachronisme ne consiste pas à projeter dans le texte occamiste le couple: usage ordinaire/usage philosophique (puisqu'il s'y trouve effectivement), mais à voir dans le premier terme un élément normatif qui servirait à mesurer la justesse du second.

Ce qui peut-être porte à confusion, c'est que, d'une part, Occam insiste fréquemment sur le caractère impropre des expressions utilisées par les philosophes et que, d'autre part, très souvent ce reproche concerne l'utilisation de ces termes abstraits que le langage ordinaire, lui, n'emploie pratiquement pas. Mais en ellesmêmes, ces considérations ne suffisent absolument pas à faire

18. S.A., pp. $42-44$.

19. Summa logicae, pars prima, chap. 8, p. $29:$ :...quamvis in vulgari locutione abstracta talia aequivalentia multis dictionibus talibus in significando raro vel numquam locum habeant, in dictis tamen philosophorum et Sanctorum frequenter inveniuntur abstracta usitata sic accipi 》. La traduction française, comme toutes celles qu'on trouvera dans cet article, est de moi. 
de notre Franciscain un "philosophe du langage ordinaire»! Il faut étudier plus en détail le fonctionnement exact de cette critique du discours abstrait et le rôle qui y est attribué à l'usage commun.

Prenons quelques exemples tirés du De Sacramento Altaris. En réponse à une série d'objections, Occam examine des propositions telles que: "generatio est in instante », «motus est in tempore , «instans est indivisibile ${ }^{20}$; et il soutient que toutes ces expressions sont improprement formées, puisque les termes generatio, instans, motus, tempus ne sont pas de véritables noms référant à des choses en soi, mais des pseudo-noms formés à partir de verbes, d'adverbes, de conjonctions, de pronoms ou de prépositions. Il oppose à ce propos (ce qui d'ailleurs peut vaguement faire penser à certaines analyses de Wittgenstein!) la phrase «generatio est in instante » à « l'eau est dans le vase ». Ce dernier énoncé affirme bel et bien qu'une chose réelle (l'eau) se trouve dans une autre chose réelle (le vase), et puisque les deux noms tiennent vraiment lieu de réalités distinctes, la phrase est correcte secundum proprietatem sermonis ${ }^{21}$. Mais dans l'autre cas, les noms utilisés (generatio et instans) ne fonctionnent pas seulement comme noms, mais comme composés d'un nom et d'un verbe: generatio désigne le fait pour quelque chose (qu'on peut nommer) d'être engendré (recours à un verbe), de sorte qu'un tel terme ne peut devenir le sujet d'une proposition que par un usage impropre $^{22}$.

Tout se passe, selon Occam ${ }^{23}$, comme si par exemple on remplaçait par décision arbitraire la locution adverbiale tunc bene par le signe $A$ et comme si on se mettait dès lors à émettre des propositions de forme : «A est quelque chose ». Puisque la phrase «tunc bene est quelque chose» est logiquement inacceptable, la phrase «A est quelque chose » devrait également être considérée comme impropre. Elle devrait par conséquent être reçue comme une expression figurée («tamquam locutio figurativa») servant

20. S.A., pp. 52 et ss.

21. Id., pp. 54-56.

22. Id., p. 42.

23. Id., p. 52. 
à remplacer quelque chose comme: «iste, qui bene tunc est, est aliquid ». De même, la proposition «generatio est in instante», lorsqu'on la trouve chez les philosophes et les saints, peut être interprétée comme une façon figurée de dire: "lorsqu'une chose est engendrée, elle n'est pas engendrée partie après partie, mais d'un seul coup ${ }^{24}$. De sorte qu'à proprement parler, les phrases du type «generatio est aliquid» ne sont ni vraies ni fausses, pas plus que des phrases comme «hominem est aliquid » où l'accusatif est indûment placé en position de sujet, ou comme «hominitas est aliquid » où bominitas serait un terme abstrait formé exclusivement à partir de l'accusatif bominem (comme generatio est formé à partir d'un verbe) ${ }^{25}$; mais elles peuvent être excusées dans la mesure où on ne les considère que comme des abréviations. L'impardonnable consiste à les prendre pour des expressions bien formées!

Toutes les analyses de ce genre chez Occam portent sur des propositions énoncées par les «philosophes» et par les «saints»:

... d'innombrables propositions du même genre que l'on trouve chez les philosophes et les saints et même dans l'Ecriture Sainte sont prises au sens figuré et doivent être expliquées par le recours à quelque figure grammaticale. ${ }^{26}$

On peut donc dire en ce sens que la critique occamiste du langage abstrait est en même temps une critique du langage philosophique et théologique et non une critique du langage ordinaire. Cela semble d'ailleurs confirmé par le fait que l'explication de l'apparition de ces modes impropres du discours ne concerne jamais que leur utilisation chez les philosophes et les saints. Ils cédaient, nous dit Occam, aux exigences soit de la versification soit de l'élégance soit de la brièveté ${ }^{27}$. Mais il ne faut pas leur en tenir rigueur puisqu’à leur époque ces procédés étaient bien

24. Id., p. 54 : «... illa propositio 'generatio est in instante', sub illo intellectu debet recipi, 'quando aliquid generatur, non generatur pars ante partem sed totum simul generatur' ».

25. Id., p. 58-60.

26. Id., p. 42 : «... et huiusmodi innumerabiles propositiones, quae inveniuntur in dictis philosophorum et sanctorum et etiam in scriptura sacra, sunt figurative per aliquam figuram grammaticalem excusandae ».

2.7. Id., p. 44 et 62 . 
connus et que tout le monde (ou en tout cas tous les sages) savait qu'il ne fallait pas prendre ces expressions au pied de la lettre ${ }^{28}$. Les philosophes modernes au contraire (ailleurs il écrit les «simples d'esprit»!) tombent dans toutes sortes d'erreurs en croyant que tous les termes abstraits comme aditas, abietas, aquietas, baectitas renvoient à des réalités distinctes ${ }^{29}$ !

Occam vise ici les scotistes, qui multipliaient à l'intérieur des êtres réels les entités formellement distinctes (en distinguant par exemple l'baeccéité du cheval de son équinité !). C'est précisément la lutte acharnée d'Occam contre cette prolifération des entités - réelles ou formelles - qui a valu à son nom d'être associé au fameux principe du rasoir. Et c'est probablement aussi cette impitoyable dénonciation des erreurs dues à un usage naîf du langage philosophique qui peut le plus porter un contemporain à confondre les positions d'Occam avec celles de G.E. Moore par exemple, pour qui selon Norman Malcolm, «tout énoncé philosophique qui fait violence au langage ordinaire est faux ${ }^{30}$, et

28. Id., p. 62.

29. Id., p. 62-64: « ... omnes tales propositiones sunt impropriae: 'aditas est aliquid', 'abietas est relatio', 'aquietas est respectus', 'haectitas est distincta a natura', et sic de multis talibus quae recipiuntur a modernis» (souligné par moi); comparer avec le passage de la p. $44:$ «...ex praedictis causis occasionnaliter multi errores proveniunt in simplicibus. quia omnia dicta philosophorum et sanctorum secundum proprietatem sermonis volunt accipere, cum tamen sint figurative sumenda » (souligné par moi).

30. «Moore's great historical role consists in the fact that he has been purhaps the first philosopher to sense that any philosophical statement wich violates ordinary language is false...», « Moore and ordinary language $\gg$ in The Pbilosophy of G. E. Moore éd. par P.A. SCHILPP, New-York, The Library of Living Philosophers, 1952, (2e édition), p. 368. Le même article est repris dans Ordinary language, éd. par V.C. CHAPPELI, New-Jersey, Prentice-Hall, 1964, p. 5-23. Jé me réfère ici à Malcolm plutôt qu'à Moore lui même parce que ce dernier n'utilise jamais de formules aussi tranchées sur la question du langage ordinaire. Par ailleurs, le problème de savoir si Moore comme philosophe correspond bien à la description qu'en donne Malcolm n'est pas ici pertinent. L'important est que Malcolm, lui, soit clairement un philosophe du langage ordinaire. Comme dit Chappell, dans son introduction (Ordinary language, p. 2) : «Malcolm may or may not be right in his interpretation of Moore (..) But his paper is valuable for its clear presentation and plausible defence of the Wittgensteinian view that philosophical problems are linguistic in character and are to be solved by appealing to the ordinary use of the expressions on wich they turn》. 
pour qui le langage ordinaire est la norme du sens propre et de l'usage correct.

Mais on peut commencer à soupçonner la gravité de cette confusion en constatant qu'en au moins une de ses rares occurrences chez Occam, la vulgaris locutio fait aussi l'objet d'une critique :

En outre, même la façon ordinaire et commune de parler utilise fréquemment de telles expressions figurées, comme en témoignent les grammairiens qui ont étudié la question. ${ }^{31}$

Si l'on se souvient que la locutio figurativa s'oppose chez Occam à la proprietas sermonis, on sera en mesure d'évaluer par cette simple citation toute la distance qui sépare notre auteur d'une position comme celle que Malcolm attribue à Moore et selon laquelle «il n'est pas possible qu'une façon ordinaire de parler soit impropre ${ }^{32}$. Chez Occam, la chose semble bel et bien possible - elle est même fréquente - et par conséquent il faut chercher ailleurs le critère de la proprietas sermonis.

\section{II — La distinction concret/abstrait et la primauté du sémantique}

On peut à cet effet reprendre les exemples de critique du langage abstrait exposés plus haut pour les analyser de plus près. On s'aperçoit alors qu'en plus de l'expression proprietas sermonis, au moins deux concepts opératoires utilisés dans cette critique demeurent encore inexpliqués: le concept de nom et le concept d'《être formé à partir de ( (fingi $a b$ ). Ces deux concepts, dis-je, sont opératoires en ce sens qu'ils permettent dans les exemples considérés de discriminer les expressions correctes des expressions impropres: la proposition «generatio est aliquid》 est figurée parce que generatio n'est pas un véritable nom et qu'il est formé

31. S.A., p. 44 : «Immo etiam frequenter vulgaris et communis modus loquendi talibus figurativis locutionibus utitur; sicut per dicta grammaticorum de talibus tractantium potest probari» (souligné par moi). Occam parle ici des expressions figurées en général et non seulement des termes abstraits. Aussi ce texte ne contredit-il pas nécessairement l'affirmation faite ailleurs (cf. note 19) selon laquelle le langage ordinaire ne recourt presque jamais aux termes abstraits.

32. «... it is not possible for an ordinary form of speech to be improper». art. cit., p. 362 . 
à partir d'un verbe. Voilà qui est difficile à interpréter en termes de recours au langage ordinaire: grammaticalement, generatio appartient bel et bien à la catégorie des substantifs, et d'autre part si l'expression fingi ab devait être reçue comme un renvoi à l'étymologie réelle, on pourrait facilement objecter à Occam que le verbe generare à son tour est formé à partir du nom genus.

En réalité, le véritable nom pour Occam se définit non pas comme catégorie grammaticale, mais comme un certain mode de signification; et de même la dépendance fingi $a b$ ne s'explique pas par une origine historique, mais par le fonctionnement interne de la signification du mot. Ainsi, discutant de la question de savoir si un point est une chose réelle, Occam écrit :

Si le mot point tenait lieu (supponat pro) de quelque chose à la façon précise d'un nom (...), il faudrait concéder qu'un point est quelque chose (aliquid) et qu'un point est une réalité (res).$^{33}$

La suite du texte révèle que finalement il n'en est rien et que le mot point correspond plutôt à un composé grammatical comme « une ligne de telle ou telle longueur ${ }^{34}$. Mais l'important pour notre propos est que cette citation nous aide à préciser le critère du véritable nom: un signe possède le statut de nom lorsqu'il tient lieu dans la proposition d'une (ou de plusieurs) chose réelle et de rien d'autre. De même fingi ab ne désigne pas d'abord une origine diachronique, mais marque plutôt la composition interne de la signification d'un mot et est indifféremment remplacé dans le texte par derivativum $a^{35}$, compositum ex ${ }^{36}$, complexum ex ${ }^{37}$, ou en forme verbale par includat ${ }^{38}$ ou aequivalet ${ }^{39}$.

C'est dans le cadre de cette redéfinition sémantique des catégories grammaticales et logiques qu'il faut comprendre toute la

33. S.A., p. 36-38: $\$$ Si punctus supponat pro aliquo ita quod habeat praecise vim nominis $(\ldots)$, debet concedi quod punctus est aliquid et quod punctus est res $»$.

34. Id., p. 38 : «hoc nomen 'punctus' aequivalebit isti, 'linea tantae vel tantae longitudinis', sive 'linea non ulterius protensa vel extensa'. 》

35. Par exemple: S.A., p. 54.

36. Par exemple: S.A., p. 38.

37. Par exemple: S.A., p. 36-38.

38. Par exemple: S.A., p. 36.

39. Par exemple: S.A., p. 38-40. 
théorie occamiste de la distinction concret/abstrait ${ }^{40}$. Au départ, cette distinction est opérée par des considérations purement morphologiques et grammaticales: un terme concret et un terme abstrait sont des noms qui commencent de la même façon, mais qui se terminent différemment comme justus et justitia, fortes et fortitudo, animal et animalitas. Généralement le terme abstrait cst plus long que le concret, et le concret peut fréquemment servir d'adjectif alors que ce n'est pas le cas pour l'abstrait ${ }^{41}$. Mais on se doute bien que de telles considérations demeurent assez superficielles aux yeux d'Occam. La véritable question qui l'intéresse concerne les différences dans les modes de signification. Et à ce propos, il énumère quatre façons de distinguer les deux catégories :

- Dans le premier cas, le concret et l'abstrait ne signifient ${ }^{42}$ pas du tout les mêmes réalités ; par exemple juste signifie un homme, mais non pas justice ${ }^{43}$.

- Dans le second cas, ils sont au contraire parfaitement synonymes; par exemple animal et animalitas réfèrent tous les deux aux mêmes individus ${ }^{44}$.

- Dans le troisième cas - le plus intéressant pour notre propos - , le terme abstrait équivaut à la signification nominale du concret plus une détermination verbale, adverbiale, prépositionnelle ou autre; par exemple bumanitas équivaut à bomme en tant qu'bomme ${ }^{45}$.

- Dans le quatrième cas, l'abstrait est un terme collectif alors que le concret ne renvoie qu'à un seul des éléments de cette collectivité; par exemple populus se dit d'un groupe et popularis peut convenir à un individu ${ }^{46}$.

40. Cf. Summa logicae, pars prima, ch. 5-9, p. $16-33$ et Quodlibeta, V, question 9.

41. Summa logicae, pars prima, ch. 5, p. 16.

42. La signification d'un terme chez Occam est la possibilité pour ce terme de tenir lieu (supponere pro) de quelque chose dans une proposition (cf. Summa logicae, pars prima, ch. 33, p. 87-88). Aussi la notion occamiste de signification fonctionne-t-elle toujours en termes référentiels: le signe «homme» signifie les hommes réels, le signe «blanc» signifie ’̀ la fois les êtres réels qui sont blancs et la blancheur comme qualité réelle.

43. Cf. Summa logicae, pars prima, ch. 5, p. 16-18.

44. Id., ch. 6-7, p. $18-27$.

45. Id., ch. 8 , p. $28-32$. 
Ceci nous permet de constater d'abord à quel point Henry se trompe en accusant Occam de réduire toutes les oppositions entre termes concrets et termes abstraits à des cas de synonymies et à une ornementation linguistique ${ }^{47}$. Le passage sur lequel il s'appuie est précisément tiré des pages consacrées au deuxième type de distinction, le seul où le concret et l'abstrait soient reconnus comme synonymes et le seul aussi qui soit considéré comme logiquement inutile ${ }^{48}$. Il y a tout de même trois autres modes de distinction dans lesquelles les termes concrets et les termes abstraits ne sont absolument pas synonymes. Et parmi ceux-là, le premier et le quatrième n'ont rien à voir avec l' «ornementation linguistique ». Le cas du troisième est un peu plus délicat et nous y reviendrons.

Deuxièmement, cette analyse de la distinction concret/abstrait montre aussi que le discours abstrait chez Occam ne se confond finalement pas avec l'ensemble du discours technique de la philosophie et de la théologie. Si en outre on tient compte du fait que les termes abstraits ne sont pas tous systématiquement dévalorisés, on ne voit plus du tout sur quoi Henry peut baser son affirmation selon laquelle «Occam (...) s'efforce continuellement de montrer l'inutilité du langage technique, ou alors il le condamne comme faux ou comme dénué de sens ${ }^{49}$; d'autant moins d'ailleurs que notre Franciscain utilise lui-même abondamment le vocabulaire technique de la philosophie aristotélicienne et de la logique terministe.

46. Id., ch. 9, p. 32-33.

47. The Logic of Saint Anselm, loc. cit., p. $26: \& \ldots$ he claims that the philosophers of antiquity would allow interpredication of abstract and concrete because the two are synonymous, their diversification arising from a desire to ornament manners of speaking, or from some equally inessential motives $»$, et p. $28:$ «... to reduce the concrete abstract distinction to a linguistic ornament (...) displays a cynical distegard for the efforts of earlier thinkers $\gg . .$.

48. Cf. Quodl. V, q. 9: «... concretum et abstractum primo, tertio et quarto modis ita conveniunt nominibus mentalibus sicut vocalibus (...) Sed concreta et abstracta secundo modo solum variant nomina vocalia et non mentalia; cuius ratio est quia impossible est quod propositio sit vera in qua ponitur illud concretum nisi illa sit vera in qua ponitur suum abstractum ». (souligné par moi) 
Finalement, et de façon plus générale, ce cadre théorique, dans lequel il est facile de situer les exemples analysés plus haut, permet d'identifier clairement la stratégie de la logique occamiste comme un dépassement systématique du niveau morphologique et syntaxique vers le niveau proprement sémantique, voire même référentiel. La classification logique ne coïncide pas avec la classification grammaticale. On se rend compte par exemple qu'en ce qui concerne le couple concret/abstrait, l'usage morphologique est trompeur puisqu'il oppose de la même façon des termes qui en réalité entretiennent entre eux des relations fort diverses. Le critère occamiste de la proprietas sermonis doit donc être cherché du côté de la théorie de la signification. La question est maintenant la suivante: comment la signification propre est-elle déterminée?

Un texte du Quodlibet $V$, question 9, nous aidera à situer le rôle exact de l'usage ordinaire dans la réponse à cette question. En parlant de la troisième façon de distinguer l'abstrait et le concret (celle qui est pertinente aux analyses du De Sacramento Altaris), Occam écrit :

Le troisième mode survient lorsque le concret et l'abstrait sont tels que les termes abstraits par convention (ad placitum instituentium) ou par l'usage des locuteurs (ex usu loquentium) inciuent quelque syncatégorême ou quelque détermination adverbiale. ${ }^{50}$

De même, dans le passage de la Summa où il étudie la même distinction, il déclare :

Les usagers (utentes) peuvent en effet, s'ils le désirent, utiliser un seul mot au lieu de plusieurs. ${ }^{51}$

Ces remarques, il faut bien le voir, n'accordent au langage ordinaire aucune espèce de priorité. D'abord l'usage dont il est ques-

49. Op. cit., p. $26:$ «... Ockham unceasingly strives to make technical language pointless, or else condemns it as false or senseless

50. Quodl. V, quest. 9: «Tertius modus est quando concretum et abstractum sic se habent quod abstracta ad placitum instituentium vel ex usu loquentium includunt aliqua sincathegoreumata vel determinationes adverbiales ...

51. Summa logicae, pars prima, ch. 8, p. 28 : « Possunt enim utentes, si voluerint, uti una dictione loco plurium $\gg$. 
tion n'est pas nécessairement l'usage ordinaire, mais n'importe quel usage conventionnel sur lequel on voudra bien s'entendre. L'exemple fourni par Occam est à cet égard significatif :

Je pourrais par exemple utiliser $A$ à la place de l'expression omnis bomo et B à la place de tantum bomo et ainsi de suite. ${ }^{\mathbf{5 2}}$

C'est ce qui explique que les philosophes anciens avaient tout à fait raison aux yeux d'Occam d'utiliser un terme comme generatio au lieu d'une longue expression, dans la mesure où la convention, bien qu'elle ne correspondît pas à la vulgaris locutio, était suffisamment connue parmi les sages. ${ }^{53}$

Ensuite - et surtout - de telles affirmations sur la convention et sur l'usage des locuteurs ne font que reculer le problème d'un cran. Si je décide d'utiliser $A$ au lieu de omnis bomo, ou generatio au lieu d'autre chose, la question devient: d'où omnis bomo tire-t-il sa signification? C'est ici qu'il faut rompre définitivement avec toute interprétation d'Occam en termes de philosophie du langage ordinaire. Dans la logique occamiste, l'explication ultime du fondement de la signification se trouve dans le recours en deça du discours conventionnel oral ou écrit à un discours naturel prélinguistique et universel.

III. - Le discours mental comme critère de la proprietas sermonis Les trois traités systématiques de logique chez Occam (par opposition aux commentaires) s'ouvrent sur une distinction des

52. Id., p. 28 : «Sicut loco istius totius 'omnis homo', possem uti hac dictione ' $A$ ', et loco istius totius 'tantum homo', possem uti hoc vocabulo 'B', et sic de aliis 》.

53. Ceci ne signifie pas pour autant, comme semble l'insinuer Trentman («Extraordinary Language and Medieval Logic», loc. cit., p. 290: cf. texte cité supra. note 8 ), que la proprietas sermonis n'est pas valorisée par Occam. Le raisonnement est le suivant: on peut, pour les raisons mentionnées, excuser les Anciens d'avoir utilisé des expressions impropres, mais il faut quand même reconnaître que chez les modernes c'est là une source d'erreurs (cf. texte cité supra, note 29) et par conséquent il est maintenant devenu préférable de les éviter autant que possible.

54. Cf. Summa logicae, pars prima, ch. 1, p. 8; «The Elementarium logicae of Ockham », éd. par E.M. BUYTAERT, Franciscan Studies, vol. 25, 1965, p. 171, et «The Tractatus logicae minor of Ockham», éd. par E.M. BUYTAERT, Franciscan Studies, vol. 24. 1964, p. 55.56. 
trois ordres du discours ${ }^{54}$ : le discours écrit, le discours oral et le discours conceptuel. Ce dernier correspond à ce qu'Augustin appelait les verba mentalia qui ne sont d'aucune langue « et qui existent seulement dans l'esprit sans pouvoir être proférés à l'extérieur ${ }^{55}$. Dans les trois cas, la relation de signification unit le signe discursif à ce dont il est appelé à tenir lieu dans la proposition ${ }^{56}$. Ainsi le mot écrit table, le vocable oral table et le concept correspondant signifient tous trois la même chose, une série d'objets réels qu'ils représentent dans leur ordre discursif respectif. Occam s'oppose sur ce point à ceux qui soutenaient, à l'instar de Thomas d'Aquin, que les signes oraux et écrits signifient les concepts. La notion occamiste de signification se rapproche ainsi davantage de la notion moderne de référence.

Mais l'important pour ce qui nous concerne est:

1) que ces trois significations correspondantes ne sont pas indépendantes l'une de l'autre, mais qu'elles sont reliées par une structure hiérarchique de dépendance, dans laquelle les termes écrits sont subordonnés (subordinata) aux termes oraux et ceux-ci aux termes conceptuels ${ }^{57}$, de sorte que :

A chaque fois que quelqu'un profère une proposition orale, il forme d'abord en son for intérieur une proposition mentale qui n'appartient à aucune langue. ${ }^{58}$

2) que la priorité du discours mental lui vient de ce que sa signification est naturelle, alors que dans les deux autres cas la signification est conventionnelle et peut varier selon les décisions ou selon l'usage des locuteurs:

L'une des différences entre ces termes est que le concept ou passion de l'esprit signifie naturellement (naturaliter) ce qu'il

55. Summa logicae, pars prima, ch. 1, p. 8-9: «Unde isti termini concepti et propositiones ex eis compositae sunt illa verba mentalia, quae beatus Augustinus $15^{\circ}$ De Trinitate dicit nullius esse linguae quae tantum in mente manent et exterius proferri non possunt $»$.

56. Id., ch. 1, p. 9 et ch. 33, p. 87-88.

57. Id., ch. 1 , p. 9.

58. Id., ch. 12 , p. 39 : «... quandocumque aliquis profert propositionem vocalem prius format interius unam propositionem mentalem, quae nullius idiomatis est $\gg$. 
signifie alors que le terme oral ou écrit ne signifie rien, si ce n'est par institution volontaire. D'où suit une seconde différence, à savoir que le terme oral ou écrit peut par convention (ad placitum) changer de signifié, alors qu'aucune convention ne peut changer le signifié d'un terme conceptuel. ${ }^{59}$

Cette théorie de la triplicité du discours et de la primauté naturelle du langage conceptuel - qui d'ailleurs sert de cadre à toute la logique occamiste - éclaire singulièrement les analyses du De Sacramento Altaris, de même que les textes consacrés à la distinction concret/abstrait. Lorsque, pour répondre aux exigences de la versification, de l'élégance ou de la brièveté, un locuteur décide de n'utiliser qu'un seul mot au lieu de plusieurs, il se trouve en réalité à remplacer une pluralité naturelle de termes conceptuels par un seul signe oral ou écrit. Ainsi pour revenir à un exemple mentionné plus haut, lorsque A se substitue à omnis bomo, il faut comprendre ce qui se passe de la façon suivante: la signification du terme conventionnel $A$ est subordonnée à la signification naturelle d'une expression mentale composée de deux concepts omnis et bomo. En d'autres termes, le locuteur forme d'abord en son for intérieur une expression mentale complexe, qu'il traduit ensuite oralement par un seul signe arbitrairement choisi par lui. C'est ce qui explique pourquoi, dans les deux passages où Occam expose la troisième façon de distinguer les termes abstraits des termes concrets ${ }^{60}$, il recourt à l'expression ad placitum instituentium - qui décrit habituellement chez lui la formation de n'importe quel signe oral ou écrit - pour rendre compte de l'apparition d'un seul terme là où secundum proprietatem sermonis il en aurait fallu plusieurs. ${ }^{61}$

59. Id., ch. 1, p. 9 : «Una (differentia) est, quod conceptus sive passio animae naturaliter significat quidquid significat; terminus autem prolatus vel scriptus nihil significat nisi secundum voluntariam institutionem. Ex quo sequitur alia differentia, videlicet, quod terminus prolatus vel scriptus ad placitum potest mutare suum significatum, terminus autem conceptus non mutat suum significatum ad placitum cujuscumque ».

60. Id., ch. 8, p. 28, et Quodl. V, quest. 9 (texte cité supra, note 50).

61. Certes Occam soutient (Quodl. v, q. 9) que le troisième mode de distinction entre le concret et l'abstrait se retrouve bel et bien - tout comme le premier et le quatrième et contrairement aux cas de synonymies - dans la structure même du discours mental. Mais ceci ne peut signifier qu'une chose : à savoir que la distinction établie conventionnellement entre un terme 
Le critère de la proprietas sermonis devient ainsi très clair : un énoncé oral ou écrit sera «propre », lorsque son agencement syntaxique (c'est-à-dire le nombre de termes utilisés et les relations logiques qu'ils entretiennent entre eux) correspondra exactement à celui de l'énoncé mental auquel il est subordonné. ${ }^{62}$ L' « usage 》 — qui d'ailleurs chez Occam est mis sur le même pied que l'invention arbitraire de signes, alors que chez les philosophes contemporains du langage ordinaire l'un et l'autre sont opposés comme le naturel à l'artificiel ${ }^{63}-n$ 'intervient que très localement pour expliquer d'une part la morphologie du signe conventionnellement choisi, et d'autre part l'écart, lorsqu'il en est un, entre la construction linguistique et la construction logique naturelle. En aucun cas, il ne peut être considéré comme normatif, puisque les locuteurs sont libres de le transformer ad placitum, et puisque de toute façon son degré de correction logique est toujours mesuré à l'étalon de ce langage intérieur universel et prélinguistique.

Plus encore: non seulement il n'est pas normatif, mais il constitue même une importante source d'erreurs logiques dans la mesure précisément où il est susceptible de s'éloigner de son modèle mental. Dans son analyse des raisonnements fautifs, Occam distingue à la suite d'Aristote et des logiciens du XIIIe siècle (sur-

concret et un terme abstrait de la troisième catégorie correspond effectivement à une différence de signification dans le discours mental. La structure de cette opposition ne peut cependant être la même dans le langage conventionnel et dans le langage mental, puisque dans le premier on n'utilise ad placitum instituentium qu'un seul terme là où la pensée conceptuelle en utilisait plusieuns.

62. C'est pourquoi Henry a tort d'affirmer que la proprietas chez Occam est clairement du côté du langage ordinaire ( $\$$... clearly lies on the side of ordinary language », op. cit., p. 26) ; et c'est pourquoi aussi Trentman a tort de lui concéder ce point (art. cit., p. 290 ; cf. texte cité supra, note 8). Certes sermo peut bien désigner ici le discours conventionnel, mais sa proprietas n'est rien d'autre que la conformité de sa structure avec la structure logique.

63. Cf. CAVELI, S. : \& Must we mean what we say ? in Ordinary language, p. 111-112: «...ordinaty langage is natural language and $(\ldots)$ its changing is natural. ( $I t$ is unfortunate that artificial language has come to seem a general alternative to natural language...) ... To see that ordinary language is natural is to see that (perhaps even see why) it is normative for what can be said». Il est évident que «naturel» ici ne s'oppose pas à «culturel 》, mais à «artificiel 》. 
tout Guillaume de Sherwood et Pierre d'Espagne), les sophismes in dictione et les sophismes extra dictionem, et il explique systématiquement les premiers par l'écart entre l'usage linguistique et la structure logique. Ainsi le sophisme d'aequivocatio est lié à l'existence dans le langage ordinaire. («in locutione communi ») aussi bien que dans le langage technique de nombreux termes équivoques ${ }^{64}$, le terme équivoque étant ailleurs défini comme «un seul signe (oral ou écrit) subordonné à plusieurs concepts ou intentions de l'esprit ${ }^{65}$. De même l'amphibologia peut survenir

"lorsqu'un énoncé signifie une chose proprie et une autre improprie, de sorte que par un tel énoncé l'une est signifiée en vertu même du discours (de virtute sermonis) et l'autre en vertu de l'intention des locuteurs (secundum intentionem loquentium). ${ }^{66}$

$\mathrm{Ce}$ dernier texte est spécialement intéressant en ce qu'il oppose explicitement le sens propre d'un énoncé à ce que les locuteurs décident (arbitrairement) d'entendre par là. Notre analyse nous permet de comprendre que le sens propre est donné par l'énoncé conceptuel dont la structure correspond ponctuellement à celle de l'énoncé oral ou écrit en question; mais par décision arbitraire - ou par inadvertance - le locuteur peut subordonner cet énoncé oral ou écrit à une séquence de concepts (ou d'intentions) dont l'agencement est différent. Cette possibilité fournit à Occam dans plusieurs autres passages la base d'une méthode de lecture des autorités ${ }^{67}$ : lorsqu'on trouve chez un auteur reconnu une proposition fausse au sens littéral (c'est-à-dire interprétée comme la reproduction exacte dans sa structure même de la proposition mentale que l'auteur avait d'abord «formée en son for intérieur »), alors il faut supposer que l'auteur, pour des raisons de versification, d'élégance ou de brièveté, s'est exprimé de façon impropre: l'agencement linguistique de son énoncé ne correspond

64. «The Tractatus logicae minor of Ockham», p. 83.

65. Summa logicae, pars prima, ch. 13, p. 41 : «...signum unum pluribus conceptibus seu intentionibus animae subordinatum $\gg$.

66. Id., p. 85 : «... quando oratio significat unum proprie et aliud improprie ita quod per talem orationem unum significatur de virtute sermonis et aliud secundum intentionem loquentium $\gg$.

67. Cf. à ce propos mon article : «Guillaume d'Occam : logique et lecture » in Critère, sept. 1972, nos 6-7, p. 95-110. 
pas vraiment à l'agencement conceptuel qu'il avait dans l'esprit. La proposition "generatio est in instante», si on la trouve chez les autorités, en constitue précisément un exemple.

\section{IV. - En guise de conclusion}

Il doit maintenant être clair que Guillaume d'Occam ne peut en aucune façon être considéré comme un philosophe du langage ordinaire, puisque son critère de la correction du discours, loin de se référer à l'usage linguistique, réside dans un discours mental non linguistique. Mais je voudrais aller encore un peu plus loin et soutenir que non seulement Occam n'est pas de fait un philosophe du langage ordinaire, mais qu'il ne peut pas l'être dans la mesure où la coupure radicale entre le conceptuel et le linguistique rend absolument impensable tout recours au langage ordinaire comme norme. Il s'agit ici de mettre au jour certains postulats implicites de la logique occamiste pour montrer comment, malgré son caractère notoirement novateur pour l'époque, elle s'inscrit très directement dans la tradition de la métaphysique occidentale, pour laquelle l'usage linguistique est toujours second par rapport à la présence intérieure de la pensée.

Mon propre discours ici n'est évidemment pas innocent. La prétention qui est la mienne de découvrir des postulats cachés relève elle-même bien sûr de certains présupposés quant à la direction dans laquelle il faut chercher ces postulats. L'hypothèse dont je pars - et que j'ai déjà exposée ailleurs ${ }^{68}$ - est que toute la métaphysique occidentale fonctionne dans une logique de l'identité qui ramène constamment le divers et le dehors à l'unité d'une présence à soi. J'ai déjà essayé de montrer plus spécialement comment sur le plan de la conceptualisation du langage cette métaphysique s'accompagnait souvent d'un privilège théori. que accordé au nom considéré comme signe isolable détenteur d'une signification propre (dans le cas d'Occam, il s'agit de sa référence à un certain nombre de substances individuelles réelles) et comme trace linguistique de l'identité ${ }^{69}$. Les textes du $D e$

68. « La métaphysique et les noms 》 in Culture et langage, éd. par J.P. BRODEUR, Montréal, Hurtubise HMH, 1973, p. 249-281.

69. C'est ce que j'ai appelé dans « $\mathrm{La}$ métaphysique et les noms», le «nominisme» que j'avais alors étudié plus spécialement chez Platon, Aris. tote, Augustin et Guillaume d'Occam. 
Sacramento Altaris que nous avons ici considérés sont à cet égard révélateurs : certains termes (generatio par exemple) y sont disqualifiés comme «figurés » parce qu'ils sont des faux noms, c'està-dire parce qu'ils ne nomment pas vraiment des individus réels. Ils doivent être décomposés en une signification nominale authentique et une signification verbale ou adverbiale, laquelle à son tour ne peut être que seconde par rapport à la désignation directe opérée par le nom ${ }^{70}$.

Mais l'important est que cette décomposition est toujours déjà réalisée dans la pensée naturelle. La purification logique de l'usage philosophique qui apparaît comme l'un des idéaux occamistes ne donne lieu ni à la construction d'un langage formel conventionnel, ni à un retour à l'usage ordinaire, mais elle consiste en un décèlement de ce qui est déjà donné - par nature dans la présence intérieure: le discours conceptuel. L'homme pense bien par nature, c'est le langage qui le corrompt! Le couple pensée/langage, comme toujours dans la métaphysique occidentale, fonctionne dans la dissymétrie, même chez un auteur habituellement classé (mais de façon impropre !) comme nominaliste. L'un des deux termes, celui qui marque l'intériorité et la présence - les termes mentaux «existent seulement dans l'esprit et ne peuvent être proférés à l'extérieur ${ }^{71}$ — par opposition à l'extériorité et à la dispersion, se voit accorder, par une procédure typique, le privilège de normer l'autre et de lui servir de critère.

On voit à quel point on se trouve ici éloigné de toute philosophie du langage ordinaire. Celle-ci en effet n'échappe peut-être pas toujours à la métaphysique ${ }^{72}$, mais sa norme en tout cas se situe résolument dans l'extériorité du langage. On y rencontre une critique continuelle de l'idée d'une pensée intérieure non verbale et universelle ${ }^{73}$, et l'on est ainsi renvoyé de l'unité du

70. Cf. Expositio super libros Peribermeneias, Bologne, 1496, ch. 2 : a de verbo ».

71. Cf. supra note 55 .

72. On n'a qu'à lire les Principia Etbica de G. E. MOORE pour s'en convaincre! (Cambridge, University Press, 1903).

73. Cf. par exemple: AUSTIN, J.L. «Are there A Priori Concepts? in Philosopbical Papers, Oxford, Oxford University Press, 1970, p. 32-54; et «The Meaning of a Word $》$, Id., p. 55-57. 
discours mental à la diversité des langues et de l'unité du concept à la diversité des mots. Le discours philosophique y fait l'objet d'une critique non pas parce qu'il s'écarte des structures de la pensée universelle, mais parce qu'il ne se conforme pas à des règles dont le caractère historique et par conséquent fluctuant est clairement reconnu ${ }^{74}$. Ainsi, s'il est vrai qu'on trouve chez Wittgenstein $^{75}$ ou chez Austin, par exemple, comme chez Occam, l'idée que les noms ne renvoient pas toujours à des réalités individuelles, jamais ces auteurs ne recourent pour expliquer ce phénomène à une distinction hiérarchisée entre les vrais noms et les, faux noms. S'il y a pour eux plusieurs catégories de noms, aucune. d'entre elles ne possède la priorité que détient chez Occam le signe mental naturel sur le signe conventionnel. La superposition en profondeur du linguistique et du conceptuel se trouve ici remplacée par la dispersion en surface de différents types de signes linguistiques. La subordination se transforme en substituabilité et la signification «naturelle» est entièrement «culturalisée ».

La philosophie du langage ordinaire présuppose ainsi à la fois le dépassement résolu de la dualité pensée/langage et la reconnaissance de l'historicité des règles discursives, deux choses qui ne pouvaient être qu'impensables à un auteur comme Guillaume d'Occam. Et c'est pourquoi en fin de compte la traduction de secundium proprietatem sermonis par «according to property of common speech » est non seulement une erreur, mais un anachronisme grave! Quant à savoir à partir de quel moment chronologique cet impensable deviendra pensable, et à quels facteurs il faut attribuer une telle mutation épistémologique, c'est là, on le conçoit aisément, une tout autre question que son importance même oblige à différer.

Université du Québec à Trois-Rivières

74. Cf. par exemple CAVELL, S. «Must we mean what we say ? (loc, cit.), p. 111-112.

75. Cf. Investigations pbilosopbiques, Trad. par P. KLOSSOWSKI, Paris, Gallimard, 1961, p. 133: «... nous nommons 'nom' des choses fort différentes; le mot 'nom' caractérise différentes sortes d'usage d'un mot, apparentées de différentes manières ». (souligné par Wittgenstein)

76. Cf. «The Meaning of a Word》, op. cit., p. 61 : «...this view that general names 'have denotation' in the same way that proper names do, is quite as odd as the view proper names 'have connotation' in the: same way that general names do...» 